\title{
Metalografía en color de los aceros inoxidables mediante la técnica de ataque coloreado $^{(\bullet)}$
}

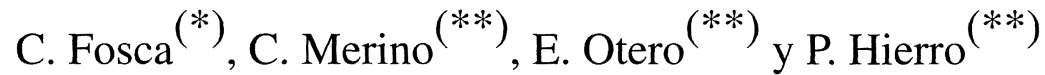 \\ Resumen La metalografía en color mediante la técnica de ataque coloreado permite la identificación y cuantifi- \\ cación, por el contraste de color, de diversas fases y constituyentes presentes en la microestructura de \\ un gran número de aleaciones. La técnica consiste en depositar una película de interferencia en la \\ superficie del material como consecuencia de reacciones electroquímicas entre el metal y el reactivo \\ de ataque coloreado. La aplicación de la técnica de ataque coloreado en los aceros inoxidables permi- \\ te la identificación y cuantificación, mediante análisis de imagen, de fases secundarias como la ferri- \\ ta en los aceros inoxidables austeníticos o la austenita secundaria y la fase sigma en los aceros inoxi- \\ dables dúplex.
}

Palabras clave: Metalografía en color. Ataque coloreado. Aceros inoxidables.

\section{Color metallography of stainless steels by tint etching}

\begin{abstract}
The color metallography by tint etching allows the identification and quantification by optical microscopy of phases and constituents present in the microstructure of a great number alloys. The principle of this technique consists of the build up of an interference film on the alloy surface as consequence of electrochemical reactions between the metallic surface and the tint etching reagent. The application of the tint etching to the metallographic analysis of stainless steels enable the identification and quantification, by image analysis, of secondary phases, as ferrite in the austenitic stainless steels, or secondary austenite and sigma phase in the duplex stainless steels.
\end{abstract}

Keywords: Color metallography. Tint etching. Stainless steels.

\section{INTRODUCCIÓN}

El ojo humano puede distinguir una increíble gama de colores. Por el contrario, su capacidad para discernir entre diferentes tonos de grises es muy limitada. Bajo esta premisa, la microestructura de un material puede lograrse, entenderse y explicarse fácilmente con el uso del color.

La morfología de los granos, representada con diferentes tonalidades de grises o simplemente reconocida a través de los límites de grano, puede revelarse y recordarse en menor grado que a través de las variaciones de color que se puedan observar

$(\bullet$ Trabajo recibido el día 11 de septiembre de 1995.

(*) Dpto. de Ingeniería Mecánica. Laboratorio de Materiales. Pontificia Universidad Católica del Perú. Av. Universitaria s/n Cdra. 18, San Miguel. Lima 32 (Perú).

(**) Dpto. de Ciencia de los Materiales e Ingeniería Metalúrgica. Facultad de Ciencias Químicas. Universidad Complutense de Madrid. 28040-Madrid (España). en cada uno de ellos. La identificación de inclusiones o fases por las diferencias de color observadas entre ellas no puede compararse de ningún modo con la representación que de las mismas pueda conseguirse a través de una variedad de grises en una fotografía en blanco y negro.

Por ello, desde hace aproximadamente 50 años, la metalografía en color se ha venido empleando para facilitar el reconocimiento de la microestructura de un material y extraer aún mayor información de ella. La primera aplicación estuvo dirigida a la identificación de inclusiones no metálicas mediante su observación empleando luz polarizada (1). Sin embargo, a lo largo de todo este tiempo, dicha técnica ha mostrado avances muy lentos debido, entre otras cosas, a los siguientes factores :

- El procesamiento de la película fotográfica en color requiere laboratorios comerciales, los cuales no están familiarizados con la fotomicrografía y pueden alterar, durante el revelado y la 
impresión, los contrastes alcanzados por la técnica metalográfica.

- Falta de apoyo financiero para la investigación en este campo.

- Falta de interés de algunos metalógrafos en emplear y desarrollar la metalografía en color en nuevos campos de aplicación.

- Los altos costos de la publicación de artículos científicos empleando fotografías en color.

Muchos de estos inconvenientes han sido salvados hoy en día y se puede decir que en los últimos 15 años la metalografía en color ha logrado considerables avances, tanto en los campos de aplicación como en el desarrollo de nuevos métodos para dar color a la imagen metalográfica. Dentro de las diferentes técnicas que conforman la metalografía en color, una de las más interesantes por su sencillez y rapidez es, sin duda, la técnica de ataque coloreado.

\section{FUNDAMENTOS DE LA TÉCNICA DE ATAQUE COLOREADO}

En estas técnicas, el color se forma por fenómenos de interferencia. Los rayos de luz que inciden sobre la superficie metálica recubierta de una película se reflejan desde ambas superficies (superficie del metal y superficie de la película), como puede apreciarse en la figura 1, con ello se obtiene un efecto de interferencia que dependerá de la longitud de onda de la luz en el aire, del espesor, $e$, y del índice de refracción de la película.

El color de la película de interferencia está relacionado con su espesor. Debido a ello, es posible obtener una amplia variedad de colores. Por ejem-

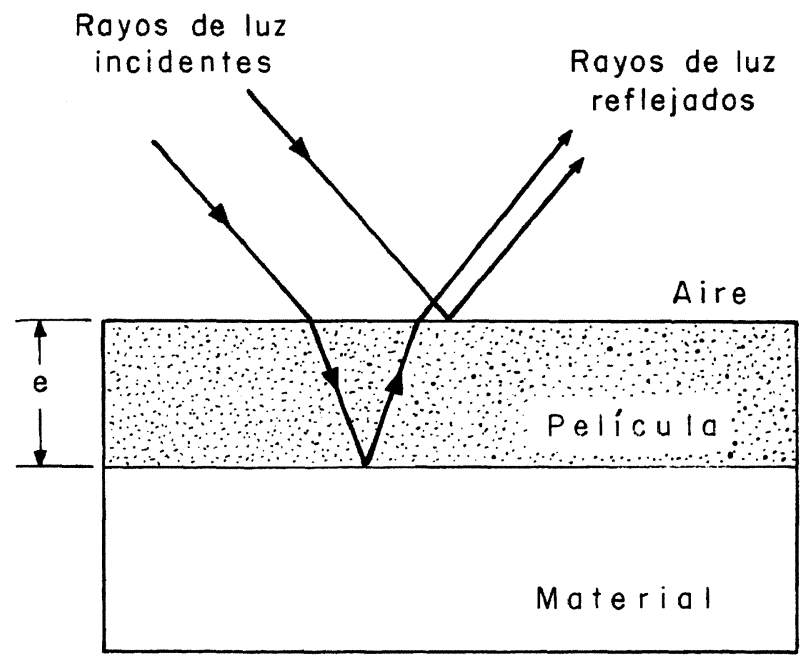

FIG. 1.- Representación esquemática del efecto de interferencia aire/película/material.

FIG. 1.- Esquematic representation of the interference phenomena in presence of a air/film/material interface. plo, cuando la película es muy delgada, la interferencia puede ocurrir en la región ultravioleta (aprox. $350 \mathrm{~nm}$ ) y no se observará color alguno. Incrementando progresivamente el espesor, la interferencia puede alcanzar la región del azul $(450 \mathrm{~nm})$, lo que significará que habrá un desfase preciso entre los rayos reflejados por la superficie metálica y por la película dentro del rango de longitudes de onda antes señalado. Ello dará lugar a que el color resultante sea el complementario del color interferido y la superficie se verá en este caso coloreada de amarillo.

El ataque coloreado es, fundamentalmente, un proceso controlado de corrosión electroquímica a través del cual se forma una película estable en la superficie de la muestra. Esta es una primera diferencia respecto a los procedimientos convencionales que emplean el ataque químico para revelar la microestructura de un material. En ellos, los productos de corrosión producidos durante el ataque se disueven en la solución y no intervienen para nada en la posterior identificación metalográfica.

En la microestructura de todos los metales y aleaciones existen zonas electroquímicamente más activas que otras, debido a que el metal a nivel microscópico presenta muchas heterogeneidades, tanto en su microestructura como en su composición química. Esto da lugar a la formación de micropilas galvánicas que crean diferencias de potencial entre los límites de grano y el interior de los mismos. De la misma forma, es posible tener diferencias en el comportamiento electroquímico entre dos granos adyacentes con diferentes orientaciones cristalográficas, o entre determinadas impurezas o compuestos intermetálicos y la matriz. Diferencias de composición química en una aleación monofásica, o presencia de varias fases en una aleación polifásica, son otras de las causas de la formación de estas diferencias de potencial electroquímico.

Durante el ataque electroquímico de una aleación bifásica, la fase más activa (anódica) resulta atacada y disuelta selectivamente, mientras que la fase más noble (catódica) permanece prácticamente inalterada.

Los reactivos de ataque coloreado son, en ese sentido, soluciones electrolíticas, normalmente de carácter ácido (disueltas en agua o alcohol), diseñadas químicamente para permitir el depósito de una fina capa (de 40 a $500 \mathrm{~nm}$ ) de óxidos, sulfuros, molibdatos complejos, selenio elemental o cromatos sobre la superficie a observar. La coloración se consigue por los fenómenos de interferencia anteriormente descritos y puede variarse fácilmente con el tiempo de ataque. La aplicación del reactivo sobre la superficie de la muestra se efectúa normalmente por inmersión. No se recomienda en estos procedimientos de ataque la aplicación del reactivo mediante algodón humedecido con la solución, 
pues tiende a producir un ataque muy desigual. La temperatura del proceso suele ser la temperatura ambiente.

En los últimos 20 años, esta técnica ha resurgido de forma muy significativa gracias al valioso aporte de una persona, que ha desarrollado la mayor cantidad de reactivos de ataque coloreado que actualmente se emplean en todo el mundo, el Sr. Emanuel Beraha, quien trabajó en los Laboratorios de la Fuerza Aérea Israelí. Es interesante hacer notar que la mayor parte de la bibliografía actual sobre reactivos de ataque coloreado hace referencia, casi de forma exclusiva, a este autor, salvo el caso de algunos otros nombres igualmente importantes como Klemm o Weck (2). Han pasado ya algunos años de la temprana muerte de este hombre, cuyo aporte en el campo de la metalografía en color ha sido enorme. Como bien refiere Yakowitz (2) "...el nombre Beraha significa en hebreo 'una bendición', y es, precisamente, lo que para muchos metalógrafos han significado los reactivos desarrollados por Beraha".

Beraha clasificó los reactivos para ataque coloreado de la siguiente manera:

a) Reactivos de carácter anódico que permiten la precipitación de una película delgada en las regiones anódicas de la microestructura, coloreando sólo estas regiones.

b) Reactivos de carácter catódico, que depositan películas de interferencia sólo sobre los constituyentes y regiones catódicas de la estructura y colorean únicamente a aquellos.

$\mathrm{Al}$ primer grupo pertenecen los reactivos de ataque elaborados con metabisulfito de potasio $\left(\mathrm{K}_{2} \mathrm{~S}_{2} \mathrm{O}_{5}\right)$, metabisulfito de sodio $\left(\mathrm{Na}_{2} \mathrm{~S}_{2} \mathrm{O}_{5}\right)$ y tiosulfato de sodio $\left(\mathrm{Na}_{2} \mathrm{~S}_{2} \mathrm{O}_{3}\right)$.

En soluciones acuosas, el $\mathrm{K}_{2} \mathrm{~S}_{2} \mathrm{O}_{5}$ se descompone en contacto con la superficie metálica, produciendo $\mathrm{SO}_{2}, \mathrm{H}_{2}$ y $\mathrm{H}_{2} \mathrm{~S}$ con un olor penetrante característico. En aleaciones pasivadas, como los aceros inoxidables, el $\mathrm{SO}_{2}$ despasiva la superficie, mientras que el $\mathrm{H}_{2} \mathrm{~S}$ proporciona los iones $\mathrm{S}^{-2}$, los cuales se combinan con los iones metálicos (de $\mathrm{Fe}$ ó $\mathrm{Ni}$ ) provenientes de la muestra para formar una película de sulfuros sobre la superficie de la aleación. La adición de $\mathrm{HCl}$ en la solución de ataque permite el empleo de este reactivo en aleaciones con alto contenido de cromo.

Beraha también desarrolló un reactivo de ataque que contenía tiosulfato, ácido cítrico y acetato de plomo (3). En este tipo de ataque, la muestra metálica actúa como catalizador y la película que se forma es sulfato de plomo ( $\mathrm{PbS})$. Se emplea mucho para el ataque coloreado de aleaciones de cobre y para identificar cementita y fosfuros en fundiciones e inclusiones no metálicas en los aceros.

$\mathrm{Al}$ segundo grupo de agentes de coloreado pertenece otro reactivo creado por Beraha, a base de ácido selénico $\left(\mathrm{H}_{2} \mathrm{SeO}_{4}\right)$, alcohol y ácido clorhídrico (4). El ácido selénico se reduce a selenio elemental y se deposita sobre las áreas catódicas de la microestructura, produciendo la coloración. El alcohol suprime la ionización y polarización en las microceldas, permitiendo, de esta manera, el depósito preferencial de la película sobre las regiones catódicas. Se emplea en la identificación de cementita en fundiciones. El principal inconveniente de este reactivo de ataque radica en que el $\mathrm{H}_{2} \mathrm{SeO}_{4}$ es muy peligroso de manipular y se requieren condiciones extremas de seguridad para su uso.

Otro agente muy popular para ataque coloreado y más seguro de usar es el reactivo a base de ácido molíbdico (desarrollado también por Beraha). Cuando una solución acuosa de $1 \% \mathrm{Na}_{2} \mathrm{MoO}_{4}$, con pequeñas adiciones de $\mathrm{HNO}_{3}$ ó $\mathrm{HCl}$ (para formar el ácido molíbdico $\mathrm{H}_{2} \mathrm{MoO}_{4}$ ), se pone en contacto con la superficie de un metal, se produce la reducción parcial del ácido, y el resultado es una coloración azulada en la solución. La solución de molibdato se reduce en la regiones de microconstituyentes catódicos depositándose en ellas una película de molibdato complejo. Cuando se combina con $\mathrm{HNO}_{3}$ la solución de $\mathrm{Na}_{2} \mathrm{MoO}_{4}$ se la emplea como reactivo de coloreado de fundiciones y aceros, y cuando se combina con $\mathrm{HCl}$ su uso se orienta principalmente a las aleaciones de aluminio. Se puede adicionar pequeñas cantidades de bifluoruro de amonio $\left(\mathrm{NH}_{4} \mathrm{FHF}\right)$ para lograr una mejor coloración en los aceros (5). En (5 y 6) se recogen listas bien documentadas de distintos reactivos para el ataque coloreado.

\subsection{Descripción de la técnica}

\subsubsection{Preparación de la muestra}

El ataque coloreado requiere una preparación muy cuidadosa de la superficie metálica. Las etapas de pulido suelen ser, normalmente, un pulido basto con pasta de diamante o alúmina $\alpha$ y un pulido final con alúmina $\gamma(0,05 \mu \mathrm{m})$. La mayor dificultad que presenta esta técnica es, sin duda alguna, el control de rayaduras en la superficie pulida, pues con el ataque coloreado se pueden detectar toda una serie de defectos de pulido que no se logra detectar con otros procedimientos de ataque metalográfico. Por ello, se recomienda muchas veces el pulido automático o electrolítico; sin embargo, esta dificultad se puede salvar con ayuda de la práctica continua y experiencia empleando incluso técnicas de pulido manual.

\subsubsection{Técnica de ataque}

Después de una adecuada preparación superficial, la muestra metalográfica debe ser limpiada 
cuidadosamente para evitar que queden partículas sobre la superficie que puedan interferir posteriormente en la deposición de la película. Se recomienda limpiar con alcohol la muestra en un baño ultrasónico e inmediatamente sumergirla en la solución de ataque coloreado. La solución se mueve ligeramente durante el período de inmersión. Durante el ataque se puede apreciar cómo la superficie va cambiando su color pasando a tonalidades como amarillo, rojo, azul y violeta. La duración del ataque suele variar entre 20 y $90 \mathrm{~s}$. Durante este intervalo de tiempo, la muestra debe permanecer sumergida y con la superficie metalográfica a la vista del metalógrafo, de manera que sea posible controlar el progreso del ataque observando los cambios en la coloración de la superficie. Una vez concluido el ataque, la muestra se extrae de la solución, se enjuaga, en agua, luego en alcohol y, finalmente, se seca, empleando aire caliente. En esta última etapa se podrá apreciar la coloración final y definitiva de la muestra metalográfica, que muchas veces suele diferir bastante de la coloración obtenida en la muestra húmeda inmediatamente después de su remoción de la solución de ataque. Los tiempos de inmersión de la muestra dependen fuertemente de la composición química del material y de su estado microestructural.

\subsubsection{Observación microscópica}

Las muestras coloreadas pueden observarse tanto en microscopios ópticos normales como de platina invertida. Estos últimos evitan mejor los posibles daños que pueda sufrir la superficie atacada al manipular la muestra en el microscopio.

Las muestras se examinan empleando normalmente iluminación normal (campo claro) y solamente filtros neutros para regular la brillantez de la imagen. También se puede emplear luz polarizada y contraste interferencial para intensificar el contraste entre fases.

El registro fotográfico puede llevarse a cabo con película fotográfica en color o en blanco y negro. Se recomienda el uso de película pancromática si se emplea ésta en blanco y negro pues las películas ortocromáticas son insensibles al color rojo. En el caso de fotografía en color, se pueden emplear distintos tipos de películas para la obtención del registro fotográfico tanto en papel como en diapositivas.

\section{METALOGRAFÍA DE ACEROS INOXIDABLES EMPLEANDO LA TÉCNICA DE ATAQUE COLOREADO}

La metalografía en color mediante la técnica de ataque coloreado tiene un campo muy interesante de aplicación en la observación microscópica de los aceros inoxidables. Su empleo no sólo está orientado a la obtención de imágenes visualmente agradables sino también, debido al elevado contraste de colores, a la identificación de fases secundarias, de zonas de material con diferencias en la composición química (segregaciones) y al empleo en la metalografía cuantitativa mediante análisis de imagen. El objetivo de este artículo es mostrar los resultados de la aplicación de esta técnica en la observación metalográfica de los aceros inoxidables.

En estos materiales, uno de los reactivos de ataque coloreado más empleado es el reactivo Beraha, que contiene $\mathrm{HCl}, \mathrm{K}_{2} \mathrm{~S}_{2} \mathrm{O}_{5}$ y $\left(\mathrm{NH}_{4} \mathrm{FHF}\right)$. Sin embargo, los autores han obtenido resultados muy buenos con la versión modificada de este agente de ataque coloreado, conocido también como reactivo de Bloech y Wedl (6), que contiene solamente $\mathrm{HCl}$ y $\mathrm{K}_{2} \mathrm{~S}_{2} \mathrm{O}_{5}$. La composición elegida del reactivo de ataque coloreado fue la siguiente:

En $100 \mathrm{ml}$ de solución:

$$
\begin{gathered}
\mathrm{HCl}(33 \%): 10-15 \mathrm{ml} \\
\mathrm{K}_{2} \mathrm{~S}_{2} \mathrm{O}_{5}: 200-500 \mathrm{mg}
\end{gathered}
$$

A fin de revelar mejor las segregaciones en estos aceros o para efectuar un macroataque en uniones soldadas o estructuras de colada, pueden emplearse mayores concentraciones $\operatorname{de}_{2} \mathrm{~S}_{2} \mathrm{O}_{5}$. Este agente químico es un reactivo de ataque coloreado de carácter anódico, que colorea intensamente las fases y regiones de material que, en contacto con esta solución, se comportan anódicamente.

La preparación del reactivo de ataque debe efectuarse instantes previos a su aplicación, pues la solución se deteriora muy rápidamente en contacto con el ambiente. El tiempo de vida útil del reactivo no suele exceder las $24 \mathrm{~h}$.

Una vez efectuado el ataque metalográfico, las muestras se enjuagaron en agua y luego se sometieron a limpieza ultrasónica en alcohol. Finalmente, se secaron en aire caliente y se procedió a su observación metalográfica.

El análisis metalográfico y posterior registro fotográfico de la microestructura coloreada se efectuó de inmediato, pues la película depositada sobre la superficie de las muestras se deteriora muy fácilmente.

En caso de que las condiciones del ataque no fueran las más adecuadas, se volvía a efectuar éste, puliendo previamente la superficie atacada con alúmina $\alpha$ y $\gamma$ sucesivamente. La película depositada se elimina fácilmente tras una corta etapa de pulido. Sólo en casos extremos, donde el ataque fue muy severo produciendo una fuerte disolución selectiva de fases presentes, se hizo necesario efectuar nuevamente la última etapa de desbaste seguido de las etapas de pulido, antes de proceder a un nuevo ataque metalográfico. 
La observación metalográfica se llevó a cabo en la sección longitudinal de cada una de las muestras, empleando un microscopio óptico Reichert MEF$4 \mathrm{M}$ de platina invertida. La observación y posterior registro fotográfico se realizó con iluminación de campo claro sin empleo de filtros de color o de polarización. El registro fotográfico se efectuó empleando una cámara fotográfica réflex de 35 $\mathrm{mm}$, acoplada al microscopio, y utilizando película en color, de $35 \mathrm{~mm}$, para luz diurna con sensibilidad ASA 100 (ISO 100). El revelado y la impresión en papel de color se realizó en un laboratorio fotográfico comercial.

La figura 2a) muestra la microestructura de un acero inoxidable austenítico, revelada mediante este agente de ataque coloreado. En ella se observan claramente los granos equiaxiales de austenita, coloreados de forma diferente, debido a su distinta orientación cristalográfica sobre el plano de observación. Se aprecia muy bien la presencia de maclas de deformación en el interior de la austenita.

Sin embargo, los aceros inoxidables austeníticos pueden presentar contenidos variables de ferrita en su microestructura como consecuencia de procesos de fabricación (aceros inoxidables moldeados) o por haber sido sometidos a operaciones de soldadura. En estas condiciones, las propiedades mecánicas y el comportamiento frente a la corrosión de estos materiales están muy influidos por la cantidad de ferrita presente en su microestructura. La técnica de ataque coloreado proporciona un elevado contraste entre la matriz austenítica y la ferrita, como puede observarse en la figura $2 \mathrm{~b}$ ) correspondiente a la microestructura de un acero inoxidable austenítico moldeado. Con el empleo de la metalografía en color, el contenido de ferrita en la microestructura puede ser estimado sin dificultad mediante análisis de imagen.

Una de las aplicaciones más interesantes de la técnica de ataque coloreado la constituyen los aceros inoxidables dúplex, debido a su estructura bifásica compuesta por cantidades aproximadamente iguales de ferrita $(\delta)$ y austenita $(\gamma)$. El reactivo empleado colorea intensa y uniformemente la ferrita cuando se analizan materiales laminados en caliente (Fig. 2c); de forma heterogénea, debido a la distinta orientación cristalográfica de los granos, cuando se observan uniones soldadas (Fig. 2d); y de forma localmente heterogénea, debido a la presencia de regiones de material con variaciones en su composición química, cuando el acero inoxidable presenta problemas de segregación. La figura 3a) muestra un claro ejemplo de ello, la microestructura corresponde a la zona central de una pieza de acero inoxidable dúplex de $40 \mathrm{~mm}$ de espesor. En ella se aprecia una región central de material, que el reactivo de ataque colorea de manera muy diferente a como lo hace en las regiones adyacentes. En esta región, la composición química de la ferrita fue diferente, observándose a mayores aumentos la presencia adicional de fases secundarias en esta fase.

La estructura bifásica de los aceros inoxidables dúplex les confiere una combinación única de elevadas propiedades mecánicas y de resistencia a la corrosión, especialmente en medios que contienen cloruros. Sin embargo, la estructura es inestable, y permanencias cortas a temperaturas entre 500 y 900 ${ }^{\circ} \mathrm{C}$ pueden provocar la transformación de la ferrita en una serie de fases secundarias que reducen drásticamente su resistencia a la corrosión y disminuyen significativamente su tenacidad (7 y 8). La identificación de fases secundarias en aceros inoxidables dúplex, mediante la técnica de ataque coloreado, ha sido empleada con mucho éxito por los autores ( 9 y 10). Empleando el reactivo descrito anteriormente, la ferrita se colorea intensamente, mientras que la austenita lo hace de forma muy ligera. En las figuras $3 b$ ) y 3d) se pueden apreciar algunas de las fases secundarias más importantes presentes en la microestructura de estas aleaciones.

Como consecuencia de enfriamientos rápidos desde temperaturas elevadas $\left(1.300{ }^{\circ} \mathrm{C}\right)$, la ferrita mantiene en solución cantidades de nitrógeno por encima de los valores termodinámicamente en equilibrio. Debido a ello, durante el enfriamiento se produce la precipitación de nitruros de cromo en el interior y en los límites de grano de la ferrita (Fig. 3b). De la misma forma, enfriamientos rápidos desde temperaturas elevadas favorecen la transformación parcial de la ferrita en austenita, en forma de granos alargados que rodean la ferrita (Fig. 3b) y/o en forma de finas placas orientadas preferentemente dentro de la matriz ferrítica (austenita Widmanstätten), como se aprecia en la figura $3 c)$. Por el contrario, enfriamientos lentos o permanencias isotérmicas a temperaturas dentro del rango de $950-650{ }^{\circ} \mathrm{C}$ pueden promover la transformación de la ferrita en otras fases secundarias, de las cuales, la fase $\sigma$ es la más importante y perjudicial, por la mayor cantidad que puede estar presente en la microestructura. Con el reactivo de ataque coloreado, la fase $\sigma$ permanece de color blanco mientras que la austenita y la ferrita se colorean creando una imagen fuertemente contrastada que permite diferenciar claramente cada una de las fases presentes.

En las figuras 3c) y $3 d$ ) se aprecian dos morfologías de la fase $\sigma$ observadas en los aceros inoxidables dúplex. En presencia de finas partículas de austenita secundaria, $\gamma_{2}$, dentro de la matriz ferrítica, la precipitación y crecimiento de la fase $\sigma$ se lleva a cabo en las interfases $\delta / \gamma$, en forma de partículas aisladas y de forma irregular (partículas de color 

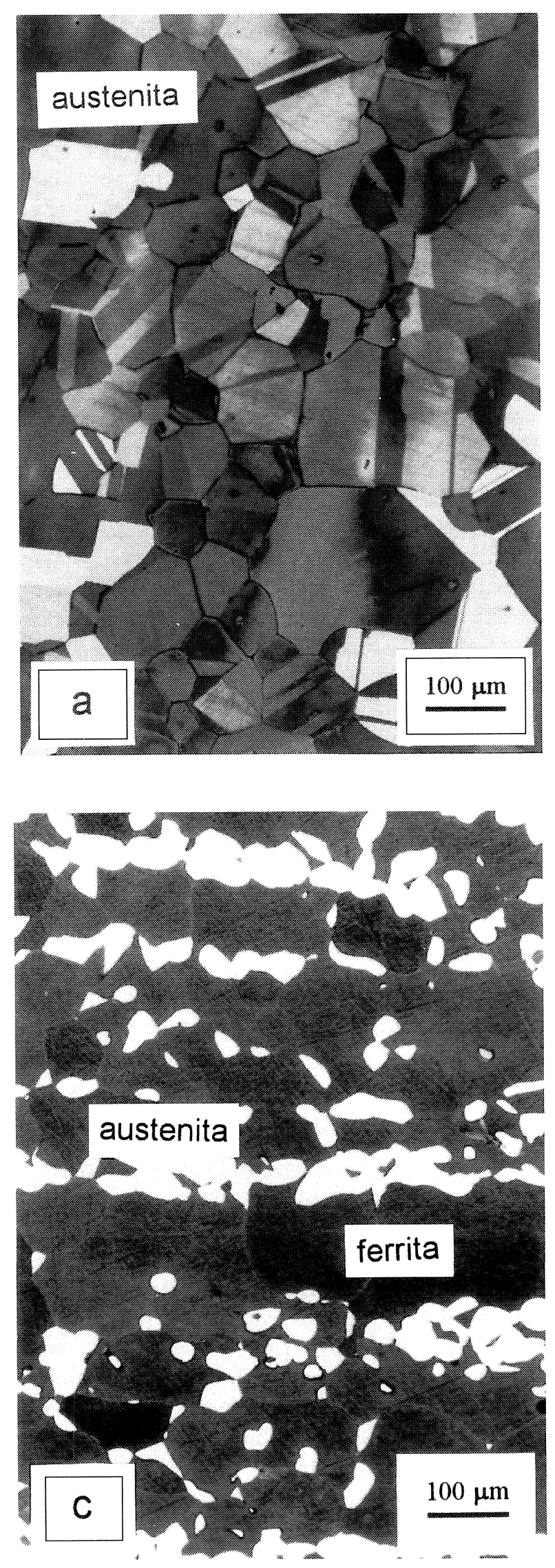
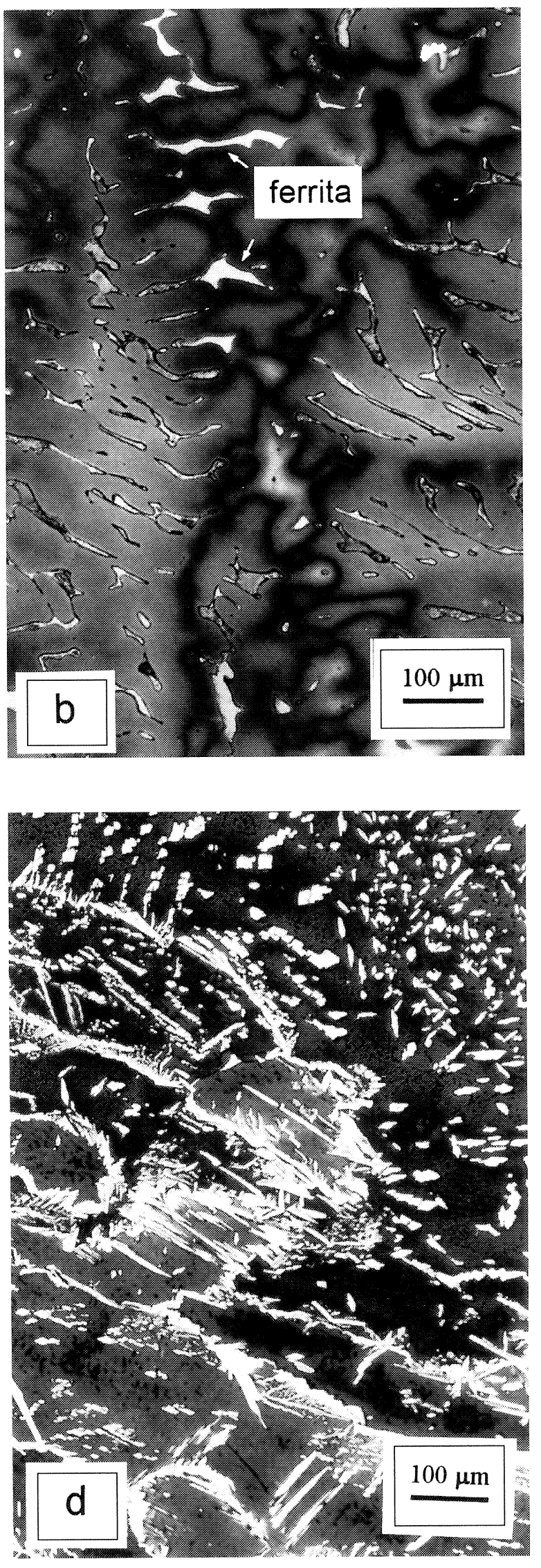

Fig. 2.- Micrografías de aceros inoxidables obtenidas mediante la técnica de ataque coloreado. FIG. 2.- Micrographs of stainless steels metallographic revealed by tint etching. 

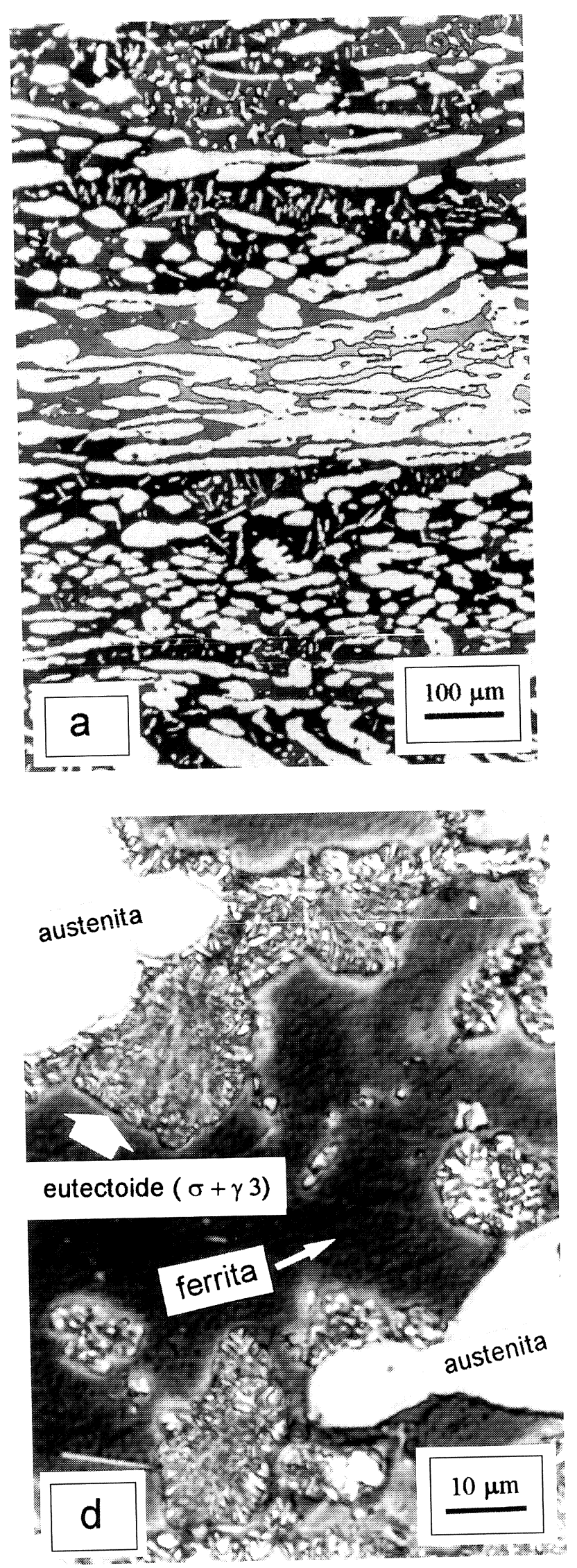
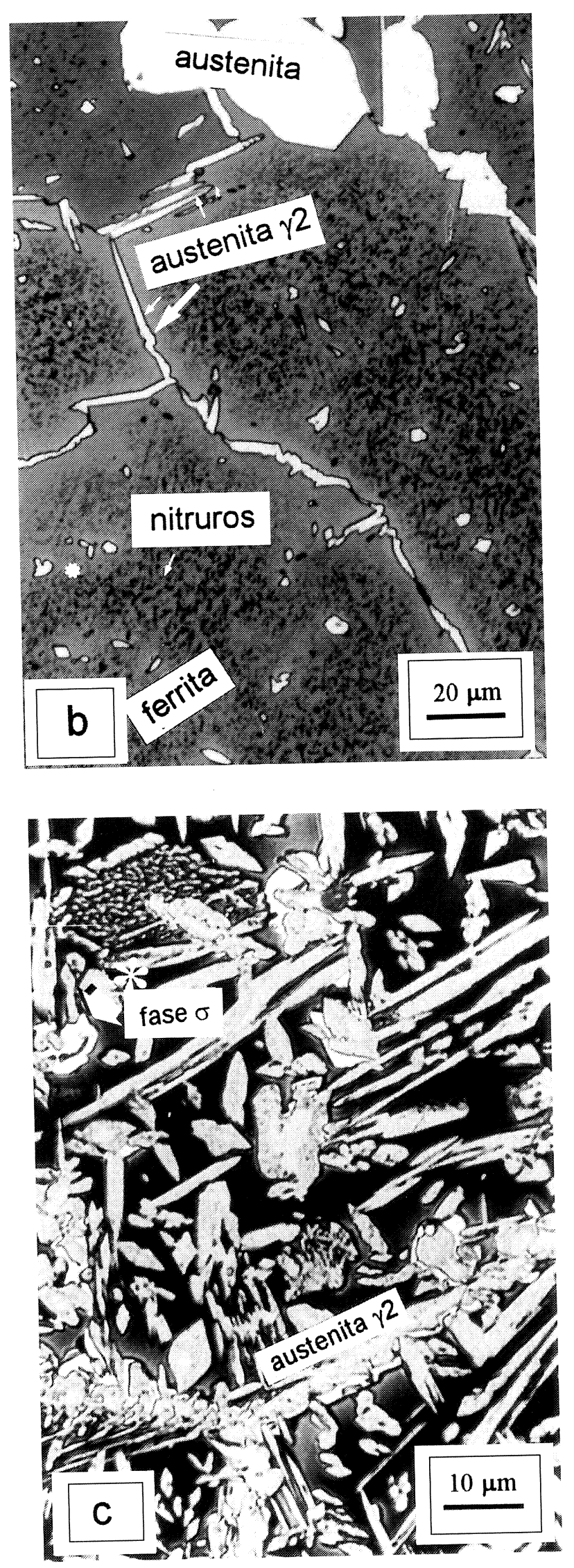

FIG. 3-Diferentes microestructuras de un acero inoxidable dúplex, que revelan la presencia de fases secundarias.

FIG. 3.-Different microstructures of a duplex stainless steel, showing the presence of secondary phases. 
blanco en la Fig. 3c). En ausencia de los precipitados de austenita $\gamma_{2}$ y cuando los granos primarios de austenita y de ferrita están relativamente distanciados, la formación de la fase $\sigma$ se produce mediante una descomposición de la ferrita que asemeja una transformación eutectoide $\delta \Rightarrow \sigma+\gamma_{3}$, con una morfología de tipo laminar y un constituyente muy similar a la perlita de los aceros al carbono (Fig. 3d).

\section{CONCLUSIONES}

El análisis metalográfico mediante microscopía óptica de los aceros inoxidables, se ve favorecido notablemente con la aplicación de la metalografía en color utilizando la técnica de ataque coloreado. Esta técnica es la más sencilla de todos los procedimientos empleados en la actualidad para "colorear" la microestructura de estas aleaciones y facilitar la identificación y/o cuantificación de fases secundarias como la ferrita en los aceros inoxidables austeníticos o la austenita $\gamma_{2}$ y la fase $\sigma$ en los aceros inoxidables dúplex. Su aplicación puede extenderse también al estudio microestructural de aceros inoxidables ferríticos y martensíticos.

\section{REFERENCIAS}

(1) Yakowitz, H. Applications of Modern Metallographic Techniques. ASTM, 1970: 49-65.

(2) WeCK, E. y LeISTNeR, E. Metallographische Anleitung zum Farbätzen nach dem Tauchverfahren. DVS. Vol. 77. 1982.

(3) VANDER Voort, G. Applied Metallography. Van Nostrand Reinhold Co. EE.UU., 1986: 7-9.

(4) VANDER VoORT, G. Metallography. Mac Graw-Hill Book Co. EE.UU., 1984: 174-182.

(5) ASM Handbook. $9^{\text {a }}$ edición, Vol. 9, ASM, EE.UU., 1985: 135-142.

(6) WeCK, E. y LeISTNER, E. Metallographische Anleitung zum Farbätzen nach dem Tauchverfahren. Parte III. DVS Vol. 77/III. 1986.

(7) Charles, J. Proc. Conf. Intern. "Duplex Stainless Steel '94”. Glasgow (Escocia), nov. 1994.

(8) Nilsson, J.-O., Wilson, A., Josefsson, B. y Thorvaldsson, T. Proc. Conf. Intern. "Applications of Stainless Steels '92”, Estocolmo (Suecia). jun. 1992.

(9) Fosca C. Proyecto de investigación. Dpto. de Ciencia de los Materiales e Ingeniería Metalúrgica. Univ. Complutense de Madrid, jun. 1993.

(10) Fosca, C., Merino, C. y Otero, E. Proc. Conf. Intern. de Metalografía "MC '95”, ASM Intern. Colmar (Francia), mayo 1995. 